\title{
Nonlinear modeling of a capacitive MEMS accelerometer using neural network
}

\begin{abstract}
This paper presents a nonlinear model for a capacitive Microelectromechanical accelerometer (MEMA). System parameters of the accelerometer are developed using the effect of cubic term of the folded-flexure spring. To solving this equation we use FEA method. The neural network (NN) uses Levenberg-Marquardt (LM) method for training the system to have more accurate response. The designed NN can identify and predict the displacement of movable mass of accelerometer. The simulation results are very promising.
\end{abstract}

